\title{
Effects of action observation therapy and mirror therapy after stroke on rehabilitation outcomes and neural mechanisms by MEG: study protocol for a randomized controlled trial
}

Tsai-yu Shih ${ }^{1}$, Ching-yi Wu ${ }^{1,2,3}$, Keh-chung Lin ${ }^{4,5}$, Chia-hsiung Cheng ${ }^{1,2,6}$, Yu-wei Hsieh ${ }^{1,2,3^{*}}$, Chia-ling Chen ${ }^{3,7}$, Chih-jou Lai ${ }^{8,9}$ and Chih-chi Chen ${ }^{3,10}$

\begin{abstract}
Background: Loss of upper-extremity motor function is one of the most debilitating deficits following stroke. Two promising treatment approaches, action observation therapy (AOT) and mirror therapy (MT), aim to enhance motor learning and promote neural reorganization in patients through different afferent inputs and patterns of visual feedback. Both approaches involve different patterns of motor observation, imitation, and execution but share some similar neural bases of the mirror neuron system. AOT and MT used in stroke rehabilitation may confer differential benefits and neural activities that remain to be determined. This clinical trial aims to investigate and compare treatment effects and neural activity changes of AOT and MT with those of the control intervention in patients with subacute stroke.

Methods/design: An estimated total of 90 patients with subacute stroke will be recruited for this study. All participants will be randomly assigned to receive AOT, MT, or control intervention for a 3-week training period (15 sessions). Outcome measurements will be taken at baseline, immediately after treatment, and at the 3-month followup. For the magnetoencephalography (MEG) study, we anticipate that we will recruit 12 to 15 patients per group. The primary outcome will be the Fugl-Meyer Assessment score. Secondary outcomes will include the modified Rankin Scale, the Box and Block Test, the ABILHAND questionnaire, the Questionnaire Upon Mental Imagery, the Functional Independence Measure, activity monitors, the Stroke Impact Scale version 3.0, and MEG signals.

Discussion: This clinical trial will provide scientific evidence of treatment effects on motor, functional outcomes, and neural activity mechanisms after AOT and MT in patients with subacute stroke. Further application and use of AOT and MT may include telerehabilitation or home-based rehabilitation through web-based or video teaching.
\end{abstract}

Trial registration: ClinicalTrials.gov, ID: NCT02871700. Registered on 1 August 2016.

Keywords: Stroke, Neurorehabilitation, Action observation, Mirror therapy, Magnetoencephalography

\footnotetext{
*Correspondence: ywhsieh@mail.cgu.edu.tw

'Department of Occupational Therapy and Graduate Institute of Behavioral Sciences, College of Medicine, Chang Gung University, 259 Wenhua 1st Rd., Guishan Dist, Taoyuan, Taiwan

${ }^{2}$ Healthy Aging Research Center, Chang Gung University, Taoyuan, Taiwan

Full list of author information is available at the end of the article
} 


\section{Background}

Stroke is the leading cause of long-term adult disability worldwide [1]. Most patients with stroke experience upperextremity (UE) motor impairment [2] and show minimal recovery of the affected arm even 6 months after stroke [3]. Due to the potentially severe adverse effects after stroke, it is critical in clinical practice to develop effective and specific stroke interventions to improve arm function and to explore the neural mechanisms involved $[4,5]$. Action observation therapy (AOT) and mirror therapy (MT) are two examples of novel approaches concerning stroke motor recovery that are supported by neuroscientific foundations $[6$, 7]. However, the relative efficacy of AOT versus MT has not been validated in patients with stroke.

AOT is a promising approach grounded in basic neuroscience and the recent discovery of the mirror neuron system (MNS) [6]. AOT commonly includes action observation and action execution and allows patients to safely practice movements and motor tasks. AOT is recommended to help patients with stroke to form accurate images of motor actions [8] and to mediate their motor relearning process after stroke [6]. Researchers have found that AOT can induce stronger cognitive activity than motor imagery in patients with stroke and have suggested that AOT could be an effective approach for patients who have difficulty with motor representation [9]. AOT is a new approach in stroke rehabilitation; therefore, only a few studies have targeted enhancement of UE motor recovery and investigated the effects of AOT in patients with stroke [8,10-14]. Based on these studies, AOT has been shown to be a beneficial and effective approach to improve patient motor function. However, the heterogeneity of study designs and small sample sizes of the studies lead to no clear conclusions about the efficacy of AOT in stroke rehabilitation.

MT has emerged as another novel stroke-rehabilitation approach during the last decade [15-17]. In this treatment, participants are instructed to move their arms and watch the action reflection of the non-affected arm in the mirror, as if it were the affected one. The process creates the visual illusion of the non-affected arm as the affected arm is normally moving. MT focuses on visual and proprioceptive feedback of the non-affected limb, which may provide substitute inputs for absent or reduced proprioceptive feedback from the affected side of the body [18]. A growing amount of academic literature has demonstrated that patients with stroke gain improvements in motor and daily function, movement control strategies, and activities of daily living $[16,17]$ after treatment with MT, which supports its use in stroke rehabilitation. In short, MT is potentially a simpler, less expensive, and effective stroke-rehabilitation approach for practical implementation in clinical settings.

Action observation is based on activities of the MNS and mainly involves brain areas of the inferior parietal lobe, inferior frontal gyrus, and ventral premotor cortex [19].
Mirror neurons discharge both during the execution of motor acts or goal-directed actions and during the observation of other people performing the same or similar actions [20]. Experimental studies in healthy adults have demonstrated that the MNS was activated during both the observation and execution of movements, which helped to form new motor patterns during action observation [21-23]. In addition, although positive effects of MT have been demonstrated in patients with stroke [24], there is no consensus about the underlying neural mechanisms of MT. Three hypotheses have been recently proposed to explain the beneficial effects of MT on motor recovery [7]. Accordingly, MT may affect perceptual motor processes via three functional neural networks: (1) activation of brain regions associated with MNS [25, 26], (2) recruitment of ipsilateral motor pathways [27], and (3) substitution of abnormal proprioception from the affected limb with feedback from the nonaffected limb $[15,18]$. Few AOT and MT neurophysiological or imaging studies have been conducted in patients with stroke. No studies have directly compared and unraveled the similarities or differences in neural plastic changes between AOT and MT in these patients. It is crucial to compare neuroplasticity mechanisms between these intervention regimens to optimize rehabilitative outcomes.

\section{Objectives}

The main purposes of this clinical trial are to (1) compare the immediate and retention treatment effects of AOT and MT on different outcomes with those of a dosematched control group and (2) explore and compare the neural mechanisms and changes in cortical neural activity associated with the effects of AOT and MT in stroke patients, using magnetoencephalography (MEG).

\section{Methods/design \\ Study design and procedure}

This study protocol conforms to the Standard Protocol Items: Recommendations for Interventional Trials (SPIRIT) guidelines (see Additional file 1). This three-arm, singleblind, randomized controlled trial will investigate the treatment effects of AOT and MT versus a control intervention and accords with the SPIRIT Figure (Fig. 1). An estimated 90 patients with subacute stroke will be recruited to participate in this study. Each participant will receive 15 training sessions for 3 weeks. Licensed occupational therapists will be well trained in treatment protocols and study procedures to ensure the consistency of intervention delivery. The therapists were not blinded to the group assignment, given the nature of the intervention. In addition, all outcome measures will be administered to the patients by the same rater, who will be blinded to the subject's group. Three evaluation time points will be used: baseline, immediately after 3 weeks of treatment, and 3 months after treatment. Prior to administration of outcome measures, a blinded 


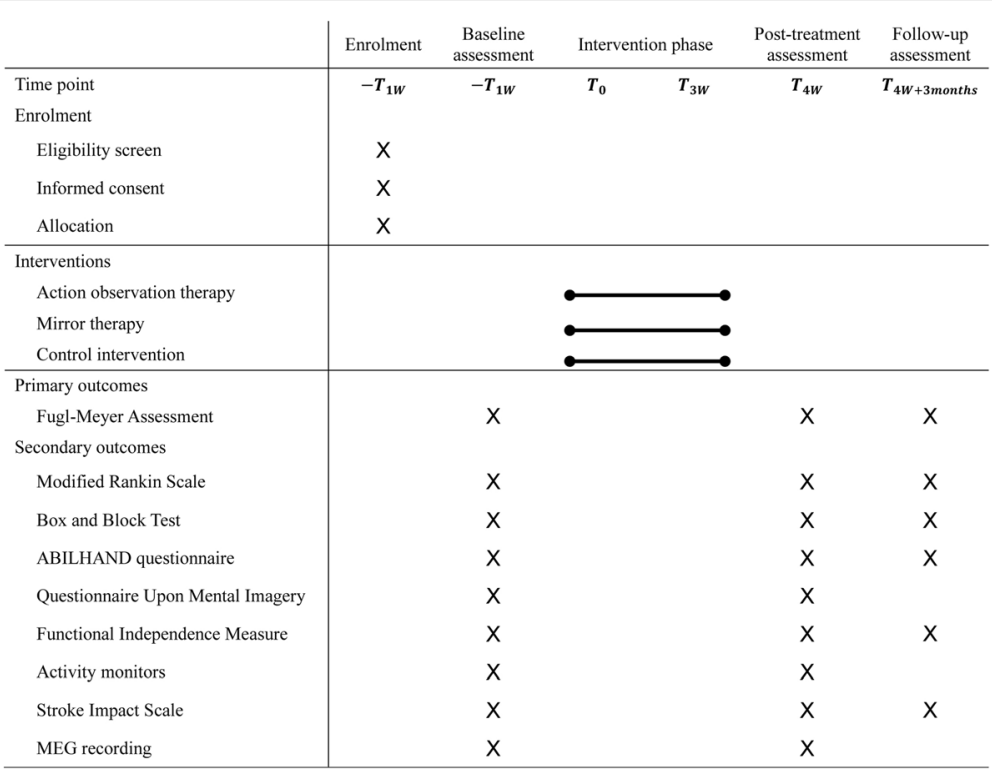

Fig. 1 Schedule of enrollment, intervention, and assessment

rater will be trained on how to appropriately administer these assessments. Rater competence will be evaluated before proceeding to the trial.

\section{Patient inclusion and exclusion criteria}

The inclusion criteria of the participants will be: (1) diagnosed as having a unilateral stroke, (2) 1 to 6 months after stroke onset, (3) from 20 to 80 years of age, (4) a baseline score of the Fugl-Meyer Assessment (FMA) of 20 to 60 [28], (5) able to follow the study instructions (measured by the Taiwan version of the Montreal Cognitive Assessment) [29], and (6) capable of participating in therapy and assessment sessions. The exclusion criteria will be: (1) global or receptive aphasia, (2) severe neglect, and (3) major medical problems or comorbidities that influenced UE usage or cause severe pain.

In addition, if patients have no metal implants, no head traumas or neurosurgical operations, and can perform MEG motor tasks, they can additionally participate in the MEG assessment. For the MEG portion of the study, we expect to recruit 12 to 15 patients in each group. Patients can still participate in the study, receiving rehabilitation therapy and clinical evaluations, even if they do not meet the MEG eligibility criteria, or are not willing to participate in the MEG study. All participants must provide written informed consent before entering the study.

\section{Randomization}

The participants will be randomly allocated to one of the three treatment groups in a 1:1:1 ratio after baseline evaluation. The participants will be pre-stratified on the basis of the severity of UE motor deficits (FMA score: 20 to 40 (moderate-to-severe) versus 40 to 60 (mild-to-moderate) [28]) and side of lesion (right versus left) to assure baseline equivalence between the groups. The random assignments will be performed online using a web-based randomization tool (freely available at https://www.randomizer.org/). A research assistant who will not be involved in outcomes assessment or screening of subjects will independently manage the randomization procedure according to allocation concealment.

\section{Intervention}

All patients will receive treatment for 60 min per day, 5 days per week for 3 weeks ( 15 sessions). For all three interventions, the therapists will provide verbal instructions, cues, feedback, and help, when needed.

\section{Action observation therapy (AOT)}

The AOT group will observe UE movement video clips and practice the movement simultaneously or sequentially. The video movements will be displayed from a first-person perspective to maximize corticomotor excitability [30]. The movements and tasks in the video will be performed by young, healthy adults, either men or women. Based on findings in the academic literature and clinical expertise, the following common categories of movements and tasks will be included in this study: (a) active range of motion (AROM) exercises (10-15 $\mathrm{min}$ ), (b) reaching movement or object manipulation (15-20 min), and (c) UE functional tasks (30 min).

During phase 1, the patients will observe AROM exercises through video clips, and move both of their UE simultaneously. In phase 2 , the patients will observe one reaching or object manipulation task, depending on the patient's motor ability, for 2 min through a video clip, and will be required to execute and practice the 
movements that they observed for $3 \mathrm{~min}$. This sequence will be repeated three times. Phase 3 will contain one functional task in each session, starting with easy then more complex tasks. Each functional task will be divided into three motor acts (Fig. 2). For example, the action of drinking bottled water will be decomposed into the following three motor acts: (1) reaching and grasping the bottle, (2) opening the bottle cap, and (3) picking up the bottle. After observing a motor act in a video clip for $2 \mathrm{~min}$, the patients will be asked to execute the actions that they observed, for $3 \mathrm{~min}$. For the next $15 \mathrm{~min}$, the patients will observe the functional task as a complete action for $2 \mathrm{~min}$ and then execute the entire task for 3 min which will be repeated three times. Examples of functional tasks are reading a magazine, folding a towel, drinking water, and wiping a table.

\section{Mirror therapy (MT)}

The MT group will receive 60 min of UE training in a mirror box. A portable and foldable mirror box that can be easily carried will be used. During the mirror practices, the patient will sit close to a table, on which the mirror box will be placed at their mid-sagittal plane. The affected arm will be placed behind the mirror, and the unaffected arm will be in front of the mirror. The patient will be instructed to watch the image of their unaffected arm in the mirror (Fig. 3). During MT, the participant will be encouraged to actively move their affected arm and hand concurrently with the mirror reflection of the unaffected arm and hand as much as they can. If necessary, the therapist will assist the participant in moving the affected hand, to synchronize the movement with the unaffected hand. MT treatment activities will also include AROM exercises (10-15 min), reaching movement or object manipulation $(15-20 \mathrm{~min})$, and functional tasks practice (30 $\mathrm{min})$ in a mirror box.

\section{Control group - customary bilateral UE training}

The control group will receive bilateral UE training without watching videos or performing activities in a mirror box. The same categories of movements and tasks as those in the AOT and MT groups will be used in the control group,

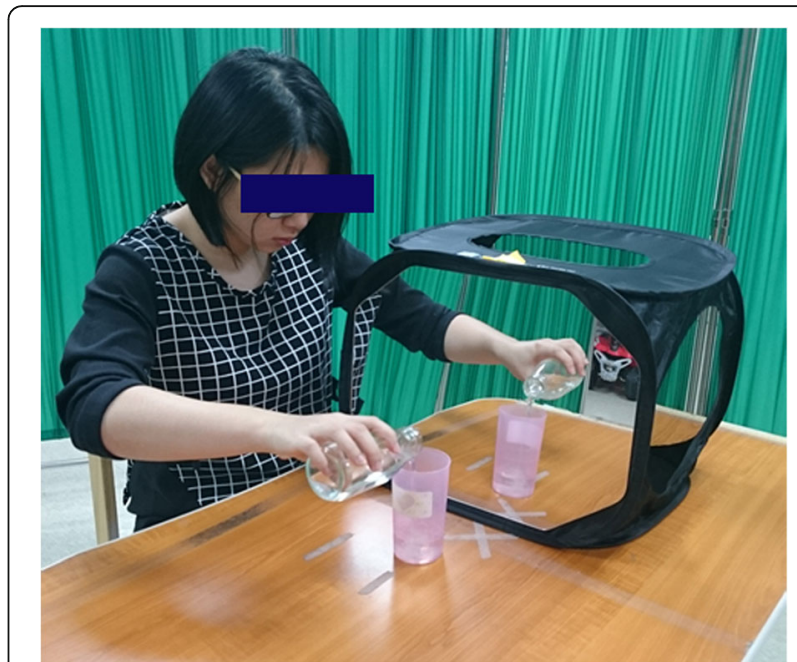

Fig. 3 Mirror therapy

but neither videos nor a mirror box will be provided. During training, the patients will be required to move both arms and hands simultaneously (Fig. 4). The levels of movement or task difficulty can be adjusted depending on the patient's level of motor ability and progress.

\section{Outcome measures}

Clinical outcome measurements will be administered to the participants at baseline, immediately after 3 weeks of treatment, and 3 months after treatment. The primary outcome will be the FMA score. Secondary outcomes will include the modified Rankin Scale (mRS), the Box and Block Test (BBT), the ABILHAND questionnaire, the Questionnaire Upon Mental Imagery (QMI), the Functional Independence Measure (FIM), activity monitors, and the Stroke Impact Scale (SIS) version 3.0.

\section{Primary outcomes}

The upper-limb subscale of the FMA will be used to evaluate motor impairments [28]. The 33 items assess movements, reflexes, and coordination of the shoulder, elbow, forearm, wrist, and hand. The total score ranges
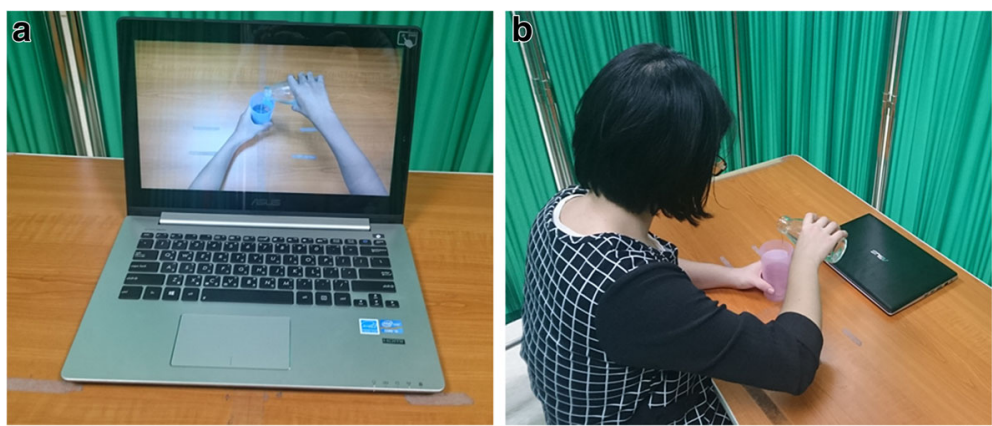

Fig. 2 Action observation therapy. a Observation of task. b Execution of task 


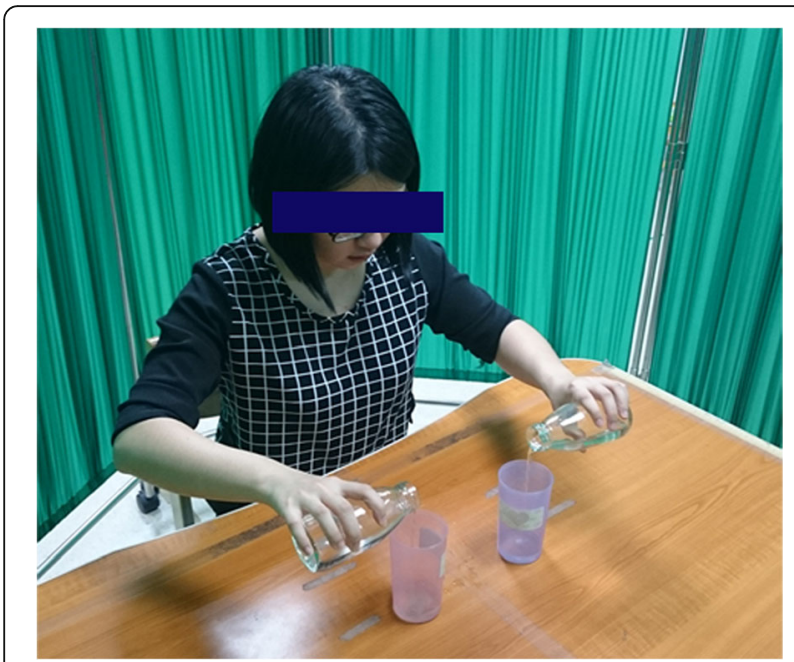

Fig. 4 Control treatment

from 0 to 66, with a higher score indicating less motor deficit. The psychometric properties of the FMA have been well established in patients with stroke [31].

\section{Secondary outcomes}

The mRS will be used to assess the degree of stroke disability in this study. The mRS was found to be valid and reliable in stroke patients [32]. The mRS scores range from 0 to 5 . Higher scores indicate more severe disability. Favorable outcomes of patients are defined as the mRS score of $\leq 2$ which indicates no or slight disability [33].

The BBT is a measure of hand dexterity with satisfactory reliability and validity in patients with stroke [34, 35]. The BBT to be used in this study contains $150 \mathrm{col}-$ ored wooden cubes in a box with two compartments. The participants will be instructed to use their affected hand to move as many blocks as possible one-by-one from one compartment to the other within $1 \mathrm{~min}$. The number of blocks transferred by the affected hand is counted as the score of the BBT.

The ABILHAND questionnaire is self-reported and assesses patients' perceived difficulty in performing daily activities that require the use of bilateral UE [36]. It contains 23 daily activities measuring bimanual ability and is rated on a 3 -point scale ( 0 = impossible, $1=$ difficult, 2 $=$ easy). Its psychometric properties have been validated in patients with stroke [37].

The short-form of the QMI will be applied to assess the ability of the patient's mental imagery. The 35-item questionnaire consists of seven domains of sensory modalities [38]. The score of each item ranges from 1 (perfectly clear) to 7 (no image present at all). A lower score indicates better mental imagery ability. The test-retest reliability of QMI has been validated [39].
The FIM is a scale frequently used to assess basic activities of daily function. It consists of 18 items with a total score ranging from 0 to 126 [40]. The FIM includes six subscales: self-care, sphincter control, transfer, locomotion, communication, and social cognition ability. A higher score indicates less disability for basic daily function. The FIM is an assessment with good reliability, validity, and responsiveness [41, 42].

The ActiGraph GT3X ${ }^{+}$accelerometers (ActiGraph, Pensacola, FL, USA) will be used to provide an objective measure of the amount of using the affected arm in the patients' real-life environments [43]. The participants will wear the accelerometers on each wrist for three consecutive days before and after treatment. The main outcome parameters will be the amount of physical activity (counts/min), activity intensity, and energy expenditure in real life. The accelerometer-based devices are commonly used to monitor physical activity in stroke patients [44].

The SIS version 3.0 is a patient-reported outcome to evaluate function, participation, and health-related quality of life of stroke survivors with sound psychometric properties [45]. It consists of 59 items, with a higher score indicating better function and greater participation.

\section{MEG recordings}

The whole-head 306-channel MEG (Vectorview, Elekta Neuromag, Helsinki, Finland) will be used in this study. The data from planar gradiometers of this device, which detect the largest signal directly above the activated cerebral areas, will be analyzed. The MEG signals will be digitized at a sampling rate of $500 \mathrm{~Hz}$, with an online bandpass of (0.1, 200) $\mathrm{Hz}$. An interval of $0.5 \mathrm{~s}$, including a pre-stimulus baseline of $0.1 \mathrm{~s}$, will be evaluated. Epochs contaminated by prominent electrooculogram signals $(>300 \mu \mathrm{V})$ and MEG artifacts $(>3000 \mathrm{fT} / \mathrm{cm}$ ) will be automatically excluded from the averaging [46]. At least 100 artifact-free evoked responses will be averaged online. For all tasks, the median nerve of the affected hand will be stimulated with an interstimulus interval of $1.5 \mathrm{~s}$ by an electrical stimulator (Konstantstrom Stimulator, Schwind, Erlangen, Germany). Stimulus intensity will be set at $20 \%$ above the motor threshold, to elicit a visible twitch of the abductor pollicis brevis muscle. In the sensory task, the subject will be asked to sit comfortably and look at a fixed position in front of them. For the motor tasks, study participants will be instructed to perform the following tasks: resting, executing movement, watching a video and executing movement simultaneously, video-watching first and then executing movement, and executing movement with and without a mirror reflection of the unaffected arm's movement. For all conditions, the beta event-related synchronization and beta event-related desynchronization will be extracted as electrophysiological indices of motor cortical activation or 
inhibition. The electromyography signals from muscles will be used to determine the onset of the movements.

\section{Monitoring potential adverse events}

Patient-reported fatigue and pain will be measured by use of an item in the Numerical Rating Scale, on a 10-cm vertical line supplemented with the Face Rating Scale. The therapist will ask the patient to rate the severity of their pain and fatigue during the treatment on a scale from 0 (no pain or no fatigue) to 10 (unbearable pain or exhaustion) on the first and final treatment days. In addition, patients will also be instructed to report any other adverse effects to the therapist or the investigator whenever they occur.

\section{Sample size calculation}

To estimate the sample size, a-priori power analysis is performed on the data from previous AOT $[8,11]$ and MT literature $[17,24]$ in stroke rehabilitation. Based on the previous data of the treatment effects on motor function outcomes (e.g., FMA), an effect size $(d)$ of 0.60 to 0.95 is expected which corresponds approximately to an effect size $(f)$ of 0.30 to 0.50 for a study design of three-group comparisons. An estimate of sample size requirements for each group in a three-group study design, given a power of 0.80 and a two-sided type-I error of 0.05 , will be in the range of 21 to 36 patients. We therefore plan to recruit 30 participants in each group (a total of 90) in this study.

\section{Data analysis}

An intention-to-treat analysis will be performed. Twoway repeated measures analysis of variance (ANOVA) will be used to evaluate the treatment effects among the three treatment groups at the three assessments from different time points. The between-subject factor will be the group and the within-subject factor will be the time. The treatment efficacy will also be examined by determining the number of participants whose change score reaches the minimal clinically important difference (MCID) in the clinical outcomes, by using a chi-square test. The MCID values will be adopted either for the established values of outcomes or $10 \%$ of the total scores when it has not been established. A $p$ value $<0.05$ will be considered as indicating statistical significance.

\section{Discussion}

The study aim is to investigate the treatment efficacy and mechanisms of neural activity of AOT and MT and compare them with those of the control intervention for patients with stroke. AOT and MT are two novel and promising approaches for enhancement of stroke motor recovery through different afferent inputs and patterns of visual feedback. AOT focuses on the observation of actual actions of another person and execution of what is seen, whereas MT emphasizes observing the mirror reflection of arm movements in the non-affected arm, as if it were the affected one. AOT and MT aim to prime the motor system through the MNS for promoting neuroplasticity and motor function of stroke patients; however, their effectiveness in stroke rehabilitation is equivocal and warrants investigation.

While translating research evidence into clinical practice, patients can receive recommended rehabilitation care and therapy. After the study has been completed, and if positive results are achieved, we will provide scientific evidence of treatment effects and neural activity changes after AOT and MT. Based on the overall study results, our approaches might become new add-on features of modern neurorehabilitative treatments, and could potentially be used in rehabilitation settings. Future applications of AOT and MT may include telerehabilitation or home-based rehabilitation through web-based or video teaching.

\section{Trial status}

At the time of submission, the recruitment of study participants is ongoing.

\section{Additional file}

Additional file 1: SPIRIT 2013 Checklist: recommended items to address in a clinical trial protocol and related documents. (PDF $60 \mathrm{~kb}$ )

\section{Abbreviations \\ AOT: Action observation therapy; AROM: Active range of motion; BBT: Box and Block Test; FIM: Functional Independence Measure; FMA: Fugl-Meyer Assessment; MCID: Minimal clinically important difference; MEG: Magnetoencephalography; MNS: Mirror neuron system; mRS: Modified Rankin Scale; MT: Mirror therapy; QMI: Questionnaire Upon Mental Imagery; SIS: Stroke Impact Scale; UE: Upper extremity}

\section{Acknowledgements}

Not applicable

\section{Funding}

This study will be supported in part by the Ministry of Science and Technology (MOST 105-2314-B-182-018) and Chang Gung Memorial Hospital (BMRPD25) in Taiwan.

\section{Availability of data and materials \\ Not applicable}

\section{Authors' contributions}

$\mathrm{YWH}$ contributed to the development of the study protocol and grant application and will contribute to project management. CYW and KCL contributed to the study design and will provide rehabilitation consultation. TYS will be involved in data collection and data management. CHC will be responsible for the MEG study and imaging processing and analysis. CLC, CJL, and CCC will provide medical consultation and subject screening and referral. TYS and YWH wrote the first draft manuscript, and all authors were involved in the revision of the manuscript. All authors have approved the final version of the article.

\section{Ethics approval and consent to participate}

This study has been approved by the Institutional Review Board of Chang Gung Medical Foundation (Approval No. 104-9173A3) in Taiwan. The study will be conducted in accordance with the Helsinki Declaration and the International Conference on Harmonization Guidelines for Good Clinical Practice. All participating therapists and evaluators will be informed of their responsibilities. 
Information about the benefits and potential risks of participation will be described in the Informed Consent Form.

\section{Consent for publication}

Volunteer consent was provided for the photographs in Figs. 2, 3, and 4 to be included in the present study.

\section{Competing interests}

The authors declare that they have no competing interests.

\section{Publisher's note}

Springer Nature remains neutral with regard to jurisdictional claims in published maps and institutional affiliations.

\begin{abstract}
Author details
${ }^{1}$ Department of Occupational Therapy and Graduate Institute of Behavioral Sciences, College of Medicine, Chang Gung University, 259 Wenhua 1st Rd., Guishan Dist, Taoyuan, Taiwan. ${ }^{2}$ Healthy Aging Research Center, Chang Gung University, Taoyuan, Taiwan. ${ }^{3}$ Department of Physical Medicine and Rehabilitation, Chang Gung Memorial Hospital, Linkou, Taiwan. ${ }^{4}$ School of Occupational Therapy, College of Medicine, National Taiwan University, Taipei, Taiwan. ${ }^{5}$ Division of Occupational Therapy, Department of Physical Medicine and Rehabilitation, National Taiwan University Hospital, Taipei, Taiwan. ${ }^{6}$ Department of Psychiatry, Chang Gung Memorial Hospital, Linkou, Taiwan. ${ }^{7}$ Graduate Institute of Early Intervention, College of Medicine, Chang Gung University, Taoyuan, Taiwan. ${ }^{8}$ Department of Physical Medicine and Rehabilitation, Taipei Veterans General Hospital, Taipei, Taiwan. ${ }^{9}$ School of Medicine, National Yang-Ming University, Taipei, Taiwan. ${ }^{10}$ College of Medicine, Chang Gung University, Taoyuan, Taiwan.
\end{abstract}

Received: 15 December 2016 Accepted: 18 September 2017 Published online: 04 October 2017

\section{References}

1. Duncan P, Studenski S, Richards L, Gollub S, Lai SM, Reker D, et al. Randomized clinical trial of therapeutic exercise in subacute stroke. Stroke. 2003;34:2173-80.

2. Hesse S, Waldner A, Mehrholz J, Tomelleri C, Pohl M, Werner C. Combined transcranial direct current stimulation and robot-assisted arm training in subacute stroke patients: an exploratory, randomized multicenter trial. Neurorehabil Neural Repair. 2011;25:838-46.

3. Kwakkel G, Kollen BJ. Predicting activities after stroke: what is clinically relevant? Int J Stroke. 2013;8:25-32.

4. Pollock A, St George B, Fenton M, Firkins L. Top ten research priorities relating to life after stroke. Lancet Neurol. 2012;11:209.

5. Dobkin BH, Carmichael ST. The specific requirements of neural repair trials for stroke. Neurorehabil Neural Repair. 2016;30:470-8.

6. Buccino G. Action observation treatment: a novel tool in neurorehabilitation. Philos Trans R Soc Lond B Biol Sci. 2014;369:20130185.

7. Deconinck FJ, Smorenburg AR, Benham A, Ledebt A, Feltham MG, Savelsbergh GJ. Reflections on mirror therapy: a systematic review of the effect of mirror visual feedback on the brain. Neurorehabil Neural Repair. 2015;29:349-61.

8. Ertelt D, Small S, Solodkin A, Dettmers C, McNamara A, Binkofski F, et al. Action observation has a positive impact on rehabilitation of motor deficits after stroke. Neuroimage. 2007;36:T164-73.

9. Kim J, Lee B, Lee HS, Shin KH, Kim MJ, Son E. Differences in brain waves of normal persons and stroke patients during action observation and motor imagery. J Phys Ther Sci. 2014;26:215-8.

10. Franceschini $M$, Ceravolo MG, Agosti M, Cavallini P, Bonassi S, Dall'Armi V, et al. Clinical relevance of action observation in upper-limb stroke rehabilitation: a possible role in recovery of functional dexterity. A randomized clinical trial. Neurorehabil Neural Repair. 2012;26:456-62.

11. Sugg K, Muller S, Winstein C, Hathorn D, Dempsey A. Does action observation training with immediate physical practice improve hemiparetic upper-limb function in chronic stroke? Neurorehabil Neural Repair. 2015;29: 807-17.

12. Kim E, Kim K. Effects of purposeful action observation on kinematic patterns of upper extremity in individuals with hemiplegia. J Phys Ther Sci. 2015;27: $1809-11$.
13. Sale P, Ceravolo MG, Franceschini M. Action observation therapy in the subacute phase promotes dexterity recovery in right-hemisphere stroke patients. Biomed Res Int. 2014;2014:457538.

14. Cha YJ, Yoo EY, Jung MY, Park SH, Park JH, Lee J. Effects of mental practice with action observation training on occupational performance after stroke. $J$ Stroke Cerebrovasc Dis. 2015;24:1405-13.

15. Altschuler EL, Wisdom SB, Stone L, Foster C, Galasko D, Llewellyn DM, et al. Rehabilitation of hemiparesis after stroke with a mirror. Lancet. 1999;353:2035-6.

16. Thieme H, Bayn M, Wurg M, Zange C, Pohl M, Behrens J. Mirror therapy for patients with severe arm paresis after stroke-a randomized controlled trial. Clin Rehabil. 2013;27:314-24.

17. Wu CY, Huang PC, Chen YT, Lin KC, Yang HW. Effects of mirror therapy on motor and sensory recovery in chronic stroke: a randomized controlled trial. Arch Phys Med Rehabil. 2013;94:1023-30.

18. Sathian K, Greenspan Al, Wolf SL. Doing it with mirrors: a case study of a nove approach to neurorehabilitation. Neurorehabil Neural Repair. 2000;14:73-6.

19. Molenberghs $P$, Cunnington $R$, Mattingley JB. Brain regions with mirror properties: a meta-analysis of 125 human fMRI studies. Neurosci Biobehav Rev. 2012;36:341-9.

20. Rizzolatti G, Craighero L. The mirror-neuron system. Annu Rev Neurosci. 2004;27:169-92.

21. Buccino G, Binkofski F, Fink GR, Fadiga L, Fogassi L, Gallese V, et al. Action observation activates premotor and parietal areas in a somatotopic manner: an fMRI study. Eur J Neurosci. 2001;13:400-4.

22. Cochin S, Barthelemy C, Roux S, Martineau J. Observation and execution of movement: similarities demonstrated by quantified electroencephalography. Eur J Neurosci. 1999;11:1839-42.

23. lacoboni M, Woods RP, Brass M, Bekkering H, Mazziotta JC, Rizzolatti G. Cortical mechanisms of human imitation. Science. 1999;286:2526-8.

24. Thieme H, Mehrholz J, Pohl M, Behrens J, Dohle C. Mirror therapy for improving motor function after stroke. Cochrane Database Syst Rev. 2012;3: CD008449.

25. Hamzei F, Lappchen CH, Glauche V, Mader I, Rijntjes M, Weiller C. Functional plasticity induced by mirror training: the mirror as the element connecting both hands to one hemisphere. Neurorehabil Neural Repair. 2012;26:484-96.

26. Matthys K, Smits M, Van der Geest JN, Van der Lugt A, Seurinck R, Stam HJ, et al. Mirror-induced visual illusion of hand movements: a functional magnetic resonance imaging study. Arch Phys Med Rehabil. 2009;90:675-81.

27. Cauraugh $\mathrm{JH}$, Summers JJ. Neural plasticity and bilateral movements: a rehabilitation approach for chronic stroke. Prog Neurobiol. 2005;75:309-20.

28. Fugl-Meyer AR, Jaasko L, Leyman I, Olsson S, Steglind S. The post-stroke hemiplegic patient. 1. a method for evaluation of physical performance. Scand J Rehabil Med. 1975;7:13-31.

29. Tsai CF, Lee WJ, Wang SJ, Shia BC, Nasreddine Z, Fuh JL. Psychometrics of the Montreal Cognitive Assessment (MoCA) and its subscales: validation of the Taiwanese version of the MoCA and an item response theory analysis. Int Psychogeriatr. 2012;24:651-8.

30. Maeda F, Kleiner-Fisman G, Pascual-Leone A. Motor facilitation while observing hand actions: specificity of the effect and role of observer's orientation. J Neurophysiol. 2002;87:1329-35.

31. Hsieh YW, Wu CY, Lin KC, Chang YF, Chen CL, Liu JS. Responsiveness and validity of three outcome measures of motor function after stroke rehabilitation. Stroke. 2009:40:1386-91.

32. Banks JL, Marotta CA. Outcomes validity and reliability of the modified Rankin scale: implications for stroke clinical trials: a literature review and synthesis. Stroke. 2007;38:1091-6.

33. Uyttenboogaart M, Stewart RE, Vroomen PC, De Keyser J, Luijckx GJ. Optimizing cutoff scores for the Barthel Index and the modified Rankin Scale for defining outcome in acute stroke trials. Stroke. 2005;36:1984-7.

34. Lin KC, Chuang LL, Wu CY, Hsieh YW, Chang WY. Responsiveness and validity of three dexterous function measures in stroke rehabilitation. J Rehabil Res Dev. 2010;47:563-71.

35. Mathiowetz V, Volland G, Kashman N, Weber K. Adult norms for the Box and Block Test of manual dexterity. Am J Occup Ther. 1985;39:386-91.

36. Penta M, Tesio L, Arnould C, Zancan A, Thonnard JL. The ABILHAND questionnaire as a measure of manual ability in chronic stroke patients: Rasch-based validation and relationship to upper limb impairment. Stroke. 2001;32:1627-34.

37. Wang TN, Lin KC, Wu CY, Chung CY, Pei YC, Teng YK. Validity, responsiveness, and clinically important difference of the ABILHAND questionnaire in patients with stroke. Arch Phys Med Rehabil. 2011;92:1086-91. 
38. Sheehan PW. A shortened form of Betts' Questionnaire upon mental imagery. J Clin Psychol. 1967;23:386-9.

39. Evans IM, Kamemoto WS. Reliability of the short form of Betts' questionnaire on mental imagery: replication. Psychol Rep. 1973;33:281-2.

40. Hamilton BB, Granger CV, Sherwin FS, Zielezny M, Tashman JS. A uniform national data system for medical rehabilitation. In: Fuhrer MJ, editor. Rehabilitation outcomes: analysis and measurement. Baltimore: Paul $\mathrm{H}$. Brookes Publishing Company; 1987. p. 137-47.

41. Beninato M, Gill-Body KM, Salles S, Stark PC, Black-Schaffer RM, Stein J. Determination of the minimal clinically important difference in the FIM instrument in patients with stroke. Arch Phys Med Rehabil. 2006;87:32-9.

42. Wallace D, Duncan PW, Lai SM. Comparison of the responsiveness of the Barthel Index and the motor component of the Functional Independence Measure in stroke: the impact of using different methods for measuring responsiveness. J Clin Epidemiol. 2002:55:922-8.

43. Domene PA, Easton C. Combined triaxial accelerometry and heart rate telemetry for the physiological characterization of Latin dance in nonprofessional adults. J Dance Med Sci. 2014;18:29-36.

44. Gebruers N, Vanroy C, Truijen S, Engelborghs S, De Deyn PP. Monitoring of physical activity after stroke: a systematic review of accelerometry-based measures. Arch Phys Med Rehabil. 2010;91:288-97.

45. Duncan PW, Bode RK, Min Lai S, Perera S. Glycine Antagonist in Neuroprotection Americans (GAIN) Investigators. Rasch analysis of a new stroke-specific outcome scale: the Stroke Impact Scale. Arch Phys Med Rehabil. 2003;84:950-63.

46. Cheng CH, Chan PY, Baillet S, Lin YY. Age-related reduced somatosensory gating is associated with altered alpha frequency desynchronization. Neural Plast. 2015;2015:302878.

\section{Submit your next manuscript to BioMed Central and we will help you at every step:}

- We accept pre-submission inquiries

- Our selector tool helps you to find the most relevant journal

- We provide round the clock customer support

- Convenient online submission

- Thorough peer review

- Inclusion in PubMed and all major indexing services

- Maximum visibility for your research

Submit your manuscript at www.biomedcentral.com/submit 